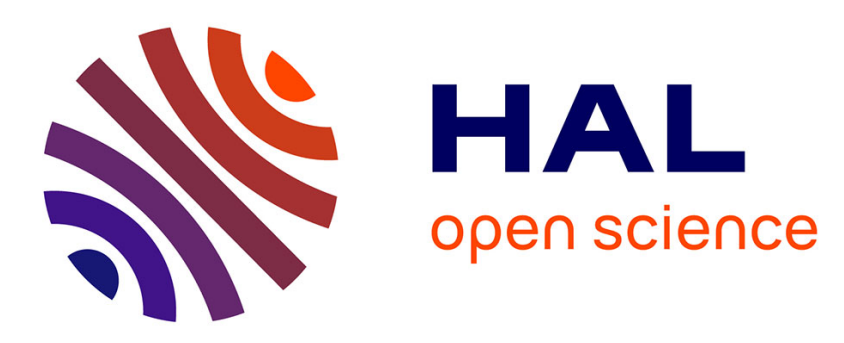

\title{
Localized proton spectroscopy without water suppression: removal of gradient induced frequency modulations by modulus signal selection.
}

Haçène Serrai, David B. Clayton, Lotfi Senhadji, Chun Zuo, Robert E. Lenkinski

\section{To cite this version:}

Haçène Serrai, David B. Clayton, Lotfi Senhadji, Chun Zuo, Robert E. Lenkinski. Localized proton spectroscopy without water suppression: removal of gradient induced frequency modulations by modulus signal selection.. J Magn Reson, 2002, 154 (1), pp.53-9. 10.1006/jmre.2001.2462 . inserm00132288

\section{HAL Id: inserm-00132288 https://www.hal.inserm.fr/inserm-00132288}

Submitted on 21 Feb 2007

HAL is a multi-disciplinary open access archive for the deposit and dissemination of scientific research documents, whether they are published or not. The documents may come from teaching and research institutions in France or abroad, or from public or private research centers.
L'archive ouverte pluridisciplinaire HAL, est destinée au dépôt et à la diffusion de documents scientifiques de niveau recherche, publiés ou non, émanant des établissements d'enseignement et de recherche français ou étrangers, des laboratoires publics ou privés. 


\title{
HAL author manuscript
}

\section{REMOVAL OF GRADIENT INDUCED FREQUENCY MODULATIONS BY MODULUS SIGNAL SELECTION.}

\author{
Haçène Serrai ${ }^{1}$, David B. Clayton ${ }^{2}$, Lotfi Senhadji ${ }^{3}$, Chun Zuo ${ }^{1}$ and Robert E. Lenkinski ${ }^{1}$ \\ ${ }^{1}$ Beth Israel Deaconess Medical Center, Harvard Medical School, Boston, MA 02215, USA. \\ ${ }^{2}$ Department of Radiology, Stanford University, Stanford, CA 94305, USA. \\ ${ }^{3}$ Laboratoire Traitement du Signal et de l'Image (LTSI), INSERM, Université de Rennes 1, \\ Rennes Cedex 35042, France.
}

Running Title: Frequency demodulations of localized MRS data.

Address Correspondence to: Lotfi Senhadji, Ph.D.,

L.T.S.I. Université de Rennes I

35042 Rennes Cedex, FRANCE

Tel : 33299281422

Fax : 33299286917

E-mail:Lotfi.Senhadji@univ-rennes1.fr 


\section{Abstract:}

Most Magnetic Resonance Spectroscopy (MRS) localization methods can generate gradient vibrations at acoustic frequencies and/or magnetic field oscillation, which can cause a time-varying magnetic field superimposed onto the static one. This effect can produce frequency modulations of the spectral resonances. When localized MRS data are acquired without water suppression, the associated frequency modulations are manifested as a manifold of spurious peaks, called sidebands, which occur symmetrically around the water resonance. These sidebands can be larger than the small metabolite resonances and can present a problem for the quantitation of the spectra, especially at short echo times. Furthermore, the resonance lineshapes may be distorted if any low frequency modulations are present. A simple solution is presented which consists of selecting the modulus of the acquired Free Induction Decay (FID) signal. Since the frequency modulations affect only the phase of the FID signal, the obtained real spectrum of the modulus is free from the spurious peaks where quantitative results may be directly obtained. Using this method, the distortions caused by the sidebands are removed. This is demonstrated by processing proton MRS spectra acquired without water suppression collected from a phantom containing metabolites at concentrations comparable to those in human brain and from a human subject using two different localization methods (PRESS and Chemical Shift Imaging PRESS-(CSI)). The results obtained illustrate the ability of this approach to remove the spurious peaks. The corrected spectra can then be fit accurately. This is confirmed by the results obtained from both the relative and the absolute metabolites concentrations in phantoms and in-vivo.

Key Words: Magnetic resonance spectroscopy; Water suppression; Frequency modulations; Localized spectroscopy. 


\section{Introduction:}

Acquisition of localized proton $\left({ }^{1} \mathrm{H}\right)$ Magnetic Resonance (MR) spectra at short echo delay without water suppression may provide several advantages compared to those acquired with water suppression (1). Preserving the water line can serve as an internal reference for metabolite absolute concentration measurements. It can also be used for corrections of lineshape distortions as well as intervoxel frequency shifts adjustments (2). However, these localized MR spectra acquired without solvent suppression can exhibit an ensemble of spurious peaks added to the metabolite resonances (3). According to the literature, the origin of these spurious peaks, called sidebands, might be the vibrations of the gradients at acoustic frequencies during data acquisition (4), the oscillation of the magnetic field ( $\left.B_{0}\right)(5)$, or a correlation between both gradient-induced acoustic vibrations and the oscillation of the $\left(B_{0}\right)$ field (6). Either one of these fluctuations induce a time-varying magnetic field, which causes a time domain signal phase contamination, manifested as signal frequency modulations. The quantification of the data obtained may be compromised if this problem is not solved. Furthermore, the lineshapes of the signal components may be distorted if the frequency modulations occur at low frequencies. The main goal of this paper is to propose a method to remove the spurious peaks from the acquired data.

Hurd et al have proposed an over-sampled PRESS-localized 2D $J$-resolved experiment to eliminate these distortions (3). However, this solution is time consuming and can limit the minimum TE possible in localized MRS techniques. A second solution, which consists of subtracting a pure water signal from the unsuppressed water data, has been proposed (4). This method may suffer from sensitivity to motion artifacts as two separate acquisitions are required. A number of gradient phase cycling schemes might also reduce these distortions, but these require at least two data acquisitions per phase cycle $(5,7)$. Post-processing methods such as the HLSVD method have been proposed (8). This approach removes only the first order harmonic of the sidebands, as they appear symmetric about the water resonance with an 
anti-phase (9). The remaining higher order harmonics may overlap with signal metabolites with small intensities.

To overcome the frequency modulations problem, we present a simple solution described as follows: Since the frequency modulations affect the phase of the Free Induction Decay (FID) signal, only the modulus of the FID signal is selected producing a real signal. As a consequence, the spurious peaks are removed from the obtained real spectrum. An additional benefit is that the lineshapes of the resonances can be corrected if low frequency modulations are present. However, as the modulus is real, the resultant spectrum is symmetrical. It includes a set of real metabolite peaks and a set of reflected peaks symmetrically located about the water resonance. Nevertheless, the metabolite resonances may be directly estimated from the spectrum of the modulus.

Here we show corrected proton phantom data and in-vivo brain spectra of a normal volunteer acquired without water suppression at short and long echo delay with PRESS and PRESS-Chemical Shift Imaging (CSI) techniques. The sidebands are removed from the spectra and the lineshapes of the resonances are corrected. To demonstrate that the proposed solution does not affect the metabolite signals and that accurate data quantitation is obtainable, the relative and absolute concentrations of the major metabolites, namely, NAA, creatine and choline of the unsuppressed water phantom and in-vivo brain data at short TE were estimated. The relative concentrations were compared to those obtained from data with water suppression. The absolute concentrations were compared to the known concentrations for the phantom and results from the literature for the in-vivo data, respectively. Good results for both relative and absolute concentrations of the metabolites were obtained from both phantom and in-vivo brain data.

Theoretically, selecting the modulus should reduce the signal-to-noise (SNR) by $\sqrt{2}$. This operation corresponds to selecting a real part of an FID signal. However, in practice we have found that the SNR is maintained and may even be increased at short TE after frequency demodulations. Possible reasons for the SNR increase observation are given in the results and discussion section. 


\section{Frequency modulations of signals in localized MR spectroscopy:}

As presented elsewhere $(4,5,6)$, the major source of the spurious peaks present throughout the spectrum at discrete frequencies is the vibration of the gradient coils at acoustic frequencies activated during their switching immediately prior to data acquisition and/or the oscillation of the magnetic field. The latter may be seen then as a superimposition of a time dependent magnetic field $B_{G}(t)$ and a static magnetic field $\left(B_{0}\right)$. This effect produces a time-varying frequency, $\omega_{G}(t)$, which produces spurious peaks with their frequencies linearly added to each metabolite resonance frequency of the signal, $\omega_{j}$ $(j=1, \ldots, N)$, where $N$ is the total number of the signal resonances.

$$
\omega_{j}+\omega_{G}(t)=\gamma\left(B_{0}\left(1-\sigma_{j}\right)+B_{G}(t)\right)
$$

with $\sigma_{j}$ denoting the shielding constant. Under these conditions an acquired FID signal takes the form:

$$
s(t)=\sum_{j=1}^{N} A_{j}(t) \cdot e^{i \cdot\left(\omega_{j} \cdot t+\varphi_{j}\right)} \cdot e^{i \cdot \omega_{G}(t)}=\sum_{j=1}^{N} s_{j}(t) \cdot e^{i \cdot \omega_{G}(t)}
$$

where $A_{j}(t)$, which may be expressed by an exponential decay, and $\varphi_{j}$ are the amplitude and the phase of the signal component $s_{j}$, respectively. The above equation represents frequency modulation of the signal induced by the term $\omega_{G}(t)$. Due to the anti-phase of the closest symmetric sidebands to the water line (first harmonic) and the phased further ones (second harmonic), the term $\omega_{G}(t)$ may be modeled as a sinusoid $(4,9)$. Furthermore, it has been shown that these spurious peaks are broad and their magnitudes decay as the echo time of the employed localization technique increases $(4,8)$. The term $\omega_{G}(t)$ may be written as:

$$
\omega_{G}(t)=A_{\mathrm{G}} \sin \left(2 \pi f_{\mathrm{G}} \cdot t+\phi_{\mathrm{G}}\right) \cdot e^{\left(-(t+T E) / T_{\mathrm{G}}\right)}
$$


where, $A_{\mathrm{G}}, f_{\mathrm{G}}, \phi_{\mathrm{G}}$ and $T_{\mathrm{G}}$ are the amplitude, the ringing frequency, phase and damping time of the gradients oscillatory vibration, respectively.

According to Eq. [2] and Eq. [3], the term $\omega_{G}(t)$ produces a group of symmetric peaks (sidebands) for each signal component. The magnitudes of these sidebands depend on the amplitude $A_{\mathrm{G}}$ as well as the amplitude $A_{j}(j=1, \ldots, N, t=0)$ of the corresponding signal component. As the strength of the magnetic field $B_{G}(t)$ is very small compared to $B_{0}$, the amplitude $A_{\mathrm{G}}$ is very small compared to $A_{j}(j=1, \ldots, N, t=0)$ and the sidebands are negligible in the localized proton spectra with water suppression. If however, the water resonance is not suppressed, the sidebands whose intensities are scaled to the water resonance become larger in intensity and may obscure the small metabolite signals. Furthermore, in the case where $f_{\mathrm{G}}$ is low $\left(f_{\mathrm{G}}<10 \mathrm{~Hz}\right.$ ), the apparent lineshape of the signal resonances may be distorted by the overlapping sidebands, which occur at low frequencies. As the frequency modulations affect only the phase of the signal, a real signal free from frequency modulations containing the major signal information may be obtained from the modulus.

Consider $s(t)$ as a noise free FID signal composed of a phased water peak and one phased metabolite resonance:

$$
s(t)=\left(A_{\mathrm{w}}(t) \cdot e^{i \cdot \omega_{\mathrm{w}} \cdot t}+A_{\mathrm{m}}(t) \cdot e^{i \cdot \omega_{\mathrm{m}} \cdot t}\right) \cdot e^{i \cdot \omega_{G}(t)}=M(t) \cdot e^{i \cdot\left(\Phi(t)+\omega_{G}(t)\right)}
$$

where $A_{\mathrm{w}}(t), A_{\mathrm{m}}(t), \omega_{\mathrm{w}}=2 \pi \delta_{\mathrm{w}}$, and $\omega_{\mathrm{m}}=2 \pi \delta_{\mathrm{m}}$ are the amplitudes and angular frequencies (chemical shift $\delta_{\mathrm{w}}, \delta_{\mathrm{m}}$ ) of water and the metabolite, respectively. The modulus $M(t)$ of the equation above is given by:

$$
M(t)=\sqrt{\left[A_{\mathrm{w}}(t) \cdot \cos \left(\omega_{\mathrm{w}} \cdot t\right)+A_{\mathrm{m}}(t) \cdot \cos \left(\omega_{\mathrm{m}} \cdot t\right)\right]^{2}+\left[A_{\mathrm{w}}(t) \cdot \sin \left(\omega_{\mathrm{w}} \cdot t\right)+A_{\mathrm{m}}(t) \cdot \sin \left(\omega_{\mathrm{m}} t\right)\right]^{2}}
$$

After some algebraic manipulations, we obtain:

$$
M(t)=\sqrt{\left[s_{r}(t)\right]^{2}+\left[A_{\mathrm{m}}(t) \cdot \sin (\Delta \omega \cdot t)\right]^{2}}
$$


where $s_{r}(t)=A_{\mathrm{w}}(t)+A_{\mathrm{m}}(t) \cdot \cos (\Delta \omega \cdot t)$ with $\Delta \omega=\omega_{\mathrm{w}}-\omega_{\mathrm{m}}$. Since the water amplitude is very large compared to the amplitude of the metabolite component, the assumption $A_{\mathrm{w}}(t)>>A_{\mathrm{m}}(t)$ holds, allowing neglecting the second term in $M(t)$. If the water peak is onresonance $\left(\omega_{\mathrm{w}}=0\right)$, the above equation may be approximated by:

$$
M(t) \approx A_{\mathrm{w}}(t)+A_{\mathrm{m}}(t) \cdot \cos \left(\omega_{\mathrm{m}} \cdot t\right)
$$

Note that in the equation above, the term $\omega_{G}(t)$ disappears. However, as the modulus is real, the resultant spectrum is symmetrical about the water resonance with a real metabolite peak and a reflected peak symmetrically located about the water resonance. To analyze the data, the high field part of the spectrum may be selected and the metabolite peak as well as the onresonance water peak may be estimated. However, as the spectrum is real, its power is divided by two, the estimated peak area of the metabolite peak must be multiplied by two. If complex data is required for analysis purpose and/or to avoid the power spectrum division as well as the symmetry of the metabolite peaks, one suggestion is to use the Hilbert Transform, which is a time-domain to a time-domain transformation (10) to produce as an output a complex signal from the input real modulus $M(t)$.

In the general case where the signal $s(t)$ contains the on-resonance water signal $\left(\omega_{\mathrm{w}}=0\right)$ and more than one metabolite component, which are not symmetric to the water resonance, the modulus is written as:

$$
M(t)=\sqrt{\left[A_{\mathrm{w}}(t)+\sum_{j=1}^{N-1} A_{j}(t) \cdot \cos \left(\omega_{j} \cdot t\right)\right]^{2}+\left[\sum_{j=1}^{N-1} A_{j}(t) \cdot \sin \left(\omega_{j} \cdot t\right)\right]^{2}+C_{r}(t)},
$$

The two remaining terms, where the second term represents the cross-terms of the metabolite components, are given by:

$$
C_{r}(t)=2 \cdot \sum_{j=1}^{N-1} \sum_{k=j+1}^{N-1} A_{j}(t) \cdot A_{k}(t) \cdot \cos \left(\omega_{k} \cdot t\right) \cdot\left[\cos \left(\left(\omega_{j}-\omega_{k}\right) t\right)-\cos \left(\omega_{j} \cdot t\right)\right]
$$


are negligible compared to the first term in $M(t)$, which may be approximated by its first term, $A_{\mathrm{w}}(t)+\sum_{j=1}^{N-1} A_{j}(t) \cdot \cos \left(\omega_{j} \cdot t\right)$. The spectrum of $M(t)$ is free from sidebands and is mainly composed of the water signal and the symmetrical metabolite resonances. The baseline distortions are corrected and the signal components recover their forms in the case of low frequency modulations. The aliphatic part of the spectrum of $M(t)$ could be selected to quantify the water and the metabolites peaks.

Note that, the static field inhomogeneities, eddy currents, etc. also induce an increase of the overlapping peaks and distortion of the spectral baseline. They affect the modulus $M(t)$ and/or the phase $\Phi(t)$ in Eq. [4] by changing the form of the envelope of the signal components from an expected exponential decay to another form. The proposed solution corrects only for the frequency modulations.

\section{Materials and Methods}

Two sets of proton spectra were collected at $127 \mathrm{MHz}$ on a 3T scanner with a maximum gradient strength of 4 gauss/cm (General Electric Medical Systems, Milwaukee, WI). The first set was collected from a $2.7 \mathrm{~L}$ spherical phantom and the second set from a healthy volunteer. Each data set contains data with and without water suppression acquired from a localized single voxel and a single slice region. PRESS and PRESS-CSI sequences were used to collect the single voxel and the 2D-CSI data, respectively. The single voxel invivo brain data were collected from white matter. The acquisition parameters were: TR $=2 \mathrm{~s}$, $\mathrm{TE}=35 \mathrm{~ms}$ and $144 \mathrm{~ms}, 32$ accumulations for the single voxel data and 1 NEX for the CSI data, $2 \times 2 \times 2 \mathrm{~cm}^{3}$ voxel size for the single voxel and $1 \times 1.5 \times 1.5 \mathrm{~cm}^{3}$ for the $2 \mathrm{D}-\mathrm{CSI}, 16 \times 16$ voxels, $24 \mathrm{~cm} \mathrm{FOV}, 5000 \mathrm{~Hz}$ spectral width, and 2K points. For the in-vivo 2D-CSI data, the spectral width was reduced to $2000 \mathrm{~Hz}$ and the data length to 512 points. For the data acquired with water suppression three CHESS pulses were added before the sequences to suppress the water resonance before data acquisition. The spherical phantom consists of $50 \mathrm{mM}$ potassium phosphate monobasic $\left(\mathrm{KH}_{2} \mathrm{PO} 4\right), 56 \mathrm{mM}$ sodium hydroxide $(\mathrm{NaOH}), 12.5 \mathrm{mM}$-acetyl-L- 
aspartic acid (NAA), $10 \mathrm{mM}$ creatine hydrate $(\mathrm{Cr}), 3 \mathrm{mM}$ choline chloride $(\mathrm{Ch}), 7.5 \mathrm{mM}$ myo-inositol (mi), $12.5 \mathrm{mM}$ L-glutamic acid (monosodium salt Glu), $5 \mathrm{mM}$ DL-lactic acid (lithium salt Lac), and $0.10 \%$ sodium azide. The modulus of each signal was obtained in order to remove the sidebands from all the data collected without water suppression and an exponential signal apodization was performed $(\mathrm{LB}=2 \mathrm{~Hz})$ before data analysis. As the magnitudes of the added sidebands are larger at short TE than longer TE (Eq. [3]), only data acquired at $\mathrm{TE}=35 \mathrm{~ms}$ are analyzed. Data acquired at TE $=144 \mathrm{~ms}$ are used to demonstrate that the phenomenon of sidebands appears at every TE but with magnitudes decreasing as the TE becomes longer. We chose to quantify data directly from the spectra of the moduli by using spectral fitting methods based upon the Levenberg-Marquardt algorithm available in a software package provided by GE (SAGE package). Only the three major metabolite peaks, namely, NAA, creatine and choline, were estimated along with the water peak in the unsuppressed water data. Absolute quantitation for the metabolites was obtained based on the water content of $110 \mathrm{M}$ for the phantom data and $70 \mathrm{M}$ for the white matter data (11). Due to the decay of the signal resonances during the TE interval, the amplitudes of the metabolites and the water peak at the origin $(t=0)$ were calculated based on the estimated amplitude values and the T1 and T2 values of the metabolites and the water given in the literature (12, 13). For the SNR calculations, the creatine resonance was selected and the SNR was calculated as (creatine peak height/"rms" noise ) x 2.5. Both data without water suppression acquired at $\mathrm{TE}=35 \mathrm{~ms}$ and $144 \mathrm{~ms}$ were used to compute the SNR and the width at half height (WHH) of the water peak before (spectrum of the original data) and after frequency demodulations (spectrum of the modulus of the original data).

\section{Results and Discussion:}

The single voxel spectra obtained from the phantom acquired at $\mathrm{TE}=35 \mathrm{~ms}$ and $\mathrm{TE}=$ $144 \mathrm{~ms}$, before and after frequency demodulations, respectively, with the water peak onresonance $\left(\omega_{\mathrm{w}}=0 \mathrm{~Hz}\right)$ are shown in Figure 1. Note that all of the spurious peaks are removed 
leading to a corrected baseline. As shown in Eq. [3], the magnitudes of the spurious peaks in the spectrum acquired at $\mathrm{TE}=35 \mathrm{~ms}$ are larger than the spectrum acquired at $\mathrm{TE}=144 \mathrm{~ms}$.

Figure 2 shows the aliphatic parts of the selected voxel spectrum obtained from the white matter of the 2D-CSI in-vivo data $(\mathrm{TE}=35 \mathrm{~ms})$ before and after frequency demodulations, respectively. The sidebands are removed from the original signal. As a consequence, the baseline is corrected and the metabolite resonances emerge, especially the NAA peak. For data without water suppression, the water peak was fit first before the metabolite resonances in the frequency domain using spectral fitting methods (Figs 3 and 4).

The results of relative and absolute concentrations for both the phantom and in-vivo data with and without water suppression acquired at $\mathrm{TE}=35 \mathrm{~ms}$ are reported in Table 1 . The fractional mean error was calculated as follows: For relative concentration the fractional mean error is the mean value of the absolute differences between both the NAA/creatine and choline/creatine ratios and their reference values divided by the reference values. For absolute concentration, the fractional mean error is the mean value of the absolute differences between the estimated concentrations of NAA, creatine and choline, and their reference values, respectively, divided by the reference ones. The reference values for both relative and absolute concentrations in the phantom are given in bold in Table 1, whereas for the in-vivo data the values given in the literature (14) are taken as reference values for absolute concentrations and given in italic bold in Table 1. The fractional mean error of the relative concentration for the phantom data was $4 \pm 2 \%$ in the unsuppressed water data and decreases to $3 \pm 1 \%$ in the suppressed results. These errors are comparable and indicate that the removal of sidebands can lead to accurate quantitation. For the in-vivo data collected with both single voxel and 2D-CSI, the values of the NAA/creatine and choline/creatine ratios in the suppressed and the unsuppressed water data are also comparable and the differences between the unsuppressed and the suppressed water data are 0.13 and 0.07 for the NAA/creatine and choline/creatine ratios, respectively. For the phantom data, the calculated fractional mean errors of absolute concentrations for NAA, creatine and choline lead to an overestimation by $7 \%, 10 \%$ and $11 \%$, respectively. These errors are comparable to those published elsewhere (15). The fractional mean errors for the absolute concentration for the in- 
vivo data are as follows: taking the results obtained by Kreis et al (14) as a reference, the NAA resonance is overestimated by approximately $10 \%$, the creatine by $6 \%$, whereas choline is underestimated by $10 \%$.

As shown above, the highest fractional mean error obtained for both relative and absolute concentrations in both data (in-vitro and in-vivo) is $11 \%$. These errors may arise from the approximation used in the modulus formula (see Eq. [7]), as well as to the quantitation method used, which is limited to one signal model, namely the lorentzian model. Other quantification methods with more flexibility on signal model may be used $(15,16)$.

It is important to note that selecting the modulus makes the lineshape of the peaks symmetric and theoretically broader than the original ones. However, as some of the modulations may occur at low frequency $\left(f_{\mathrm{G}}<10 \mathrm{~Hz}\right)$, the resulting sideband peaks resonating close the metabolite peaks including the water resonance are added to the metabolite peaks and may make them appear broader than they should. In this case, the technique described here will remove all of the sidebands, which may lead to narrower resonances in the spectrum (Table 2).

Selecting the modulus of an FID signal should decrease its SNR by $\sqrt{2}$, which corresponds to selecting the real part of an FID. However, in this case, as the spurious peaks generated by the frequency modulations are added throughout the spectrum, their higher order harmonics at high frequencies decrease in magnitudes and may be added to the "pure" noise of the signal in the noise region leading to an overestimation of the "noise", especially at short TE. As a consequence, the SNR may decrease in the data without water suppression before frequency demodulations. The use of modulus may improve the SNR because:

i) The removal of the modulations will lead to a "purer" representation of the noise which should lower the estimates of the "rms" noise level;

ii) The resulting metabolite peak in this region may be sharper since its corresponding sidebands due to the low frequency modulations are removed.

The results reported in Table 2 of the calculated SNR for creatine serve to illustrate these hypotheses. The differences between the SNR of data without water suppression after frequency demodulations and before frequency demodulations are always higher at short TE 
than longer TE. A possible explanation of this observation is that the magnitudes of spurious peaks are higher at short TE than long TE as stated by Eq. [3]. As a consequence, the "noise" corrupted by higher order harmonics of the sidebands in proton data without water suppression is higher at short TE than long TE. At TE $=144 \mathrm{~ms}$, the SNR of the corrected data after the removal of the spurious peaks is still slightly better than the SNR of data before correction. One possible explanation for this second observation is that even if the SNR is reduced by $\sqrt{2}$ in the modulus, the removal of sidebands from the metabolite resonances as well as the noise region is still sufficient for the SNR to be slightly higher in the corrected spectra (after frequency demodulations) than the uncorrected ones (before frequency demodulations).

A consequence of the proposed method is that if different metabolite peaks occur at symmetric frequencies about the water resonance in the original spectrum, they will be added and appear at the same frequency after selecting the modulus. Peaks with small amplitudes between 6 and 8 ppm have been observed in the proton spectra of brain (17). The use of the modulus will reflect these observed peaks in the NAA/glutamate region. However, in order to optimize the SNR in the acquired data, the bandwidths of the spatially selective PRESS sequence used in the GE scanner are centered on the creatine/choline region and set to about $600 \mathrm{~Hz}$ in the spectral dimension. We have experimentally observed that using this sequence, the amplitudes of peaks at 6-8 ppm are attenuated by about $75 \%$. Thus, the effect of these reflected peaks on the NAA/glutamate region after selecting the modulus are probably insignificant.

\section{Conclusions:}

We have described a method for removing spurious peaks due to the frequency modulations induced by the gradient vibrations at acoustic frequencies in localized proton MRS data without suppression of water. The method is based on selecting the modulus of the MRS signal. The results obtained show that any baseline distortions and signal component 
lineshape deformations caused by the presence of the spurious peaks are corrected in localized MRS spectra acquired without water suppression at short TE.

We have also demonstrated that after frequency demodulations the metabolite signals can be fit, and their absolute concentrations can be obtained using the water peak as an internal reference. In the absolute quantitation we concentrated on the principal metabolite peaks, NAA, creatine and choline. This quantitation can be extended to other metabolites with smaller concentrations and closer to the water peak may be added to illustrate the feasibility of the approach. However, as the main target of this work is to demonstrate that selecting the modulus is sufficient to remove the spurious peaks and to preserve the metabolite signals, no attempt was made to analyze other metabolite resonances. Furthermore, the proposed method is independent of the acquisition parameters, especially the echo time (TE). Although the use of the modulus should, in principle, reduce the SNR by $\sqrt{2}$ and lead to broader resonances, in practice we found that the SNR is maintained or increased and the resonance linewidths are retained. The method is straightforward, computationally rapid, and easily implemented in the analysis of in-vivo single voxel and multi-voxel CSI data. 


\section{References:}

1. J.W.C. Van der Veen, D.R. Weinberger, G. Tedeschi, J.A. Franck, and J.H. Duyn, Proton MR spectroscopic imaging without water suppression, Radiology, 217(1), 296-300 (2000).

2. D. B. Clayton, M.A. Elliott, and R.E. Lenkinski, In vivo proton spectroscopy without solvent suppression, Conc. Magn. Reson. 13(4), 260-275 (2001).

3. R.E. Hurd, D. Gurr, and N. Sailasuta, Proton spectroscopy without water suppression: The oversampled J-resolved experiment, Magn. Reson. Med. 40, 343-347 (1998).

4. D.B. Clayton, M.A. Elliott, J.S. Leigh, and R.E. Lenkinski, Characterization of gradientinduced signal modulations: Implications for proton MR spectroscopy methods, Proceedings of the VIII ISMRM Meeting, Glasgow, April, p.1686 (2001).

5. L.N. Ryner, P. Stroman, T. Wessel, D. I. Hoult, and J.K. Saunders, Effect of oscillatory eddy currents on MR spectroscopy, Proceedings of the VI ISMRM Meeting, Sydney, April, Vol. 3, p.1903 (1998).

6. Y. Wu, B.A. Chronik, C. Bowen, C.K. Mechefske, and B.K. Rutt, Gradient-induced acoustic and magnetic field fluctuations in a 4T whole-body MR imager, Magn. Reson. Med. 44, 532-536 (2000).

7. R. Kreis, and C. Boesch, Localized 1H-MRS without water saturation: Techniques and initial results for human brain and muscle, Proceeding of the VI ISMRM meeting, Sydney April, p.24 (1998).

8. M.A. Elliott, D.B. Clayton, and R.E. Lenkinski, 1H spectroscopy without water suppression: Removal of sidebands modulations at short TE, Proceedings of the VIII ISMRM Meeting, Glasgow, April, p.1667 (2001). 
9. H.S. Black, Modulation theory, Van Nostrand Compagny, Inc. New York (1953).

10. J.S. Bendat, and A.G. Piersol, Random data: Analysis and measurement procedures, New York Wiley J. (1986).

11. T. Ernst, R. Kreis, and B.D. Ross, Absolute quantitation of water and metabolites in the human brain. II. Compartments and water, J. Magn. Reson. B, 102, 1-8 (1993).

12. S.M. Noworolski, S.J. Nelson, R.G. Henry, M.R. Day, L.L. Wald, J. Star-Lack, and D.B. Vigneron, High spatial resolution 1H-MRSI and segmented MRI of cortical gray matter and subcortical white matter in three regions of the human brain, Magn. Reson. Med. 41, 21-29 (1999).

13. P.A. Bottomley, T.H. Foster, R.E. Argersinger, and L.M. Pfeifer, A review of normal tissue hydrogen NMR relaxation times and relaxation mechanism from 1-100 MHz: Dependence on tissue type, NMR frequency, temperature, species, excision, and age, Med. Phys. 11(4), 425-448 (1984).

14. R. Kreis, T. Ernst, and B.D. Ross, Absolute quantitation of water and metabolites in the human brain. II. Metabolite concentrations, J. Magn. Reson. B, 102, 9-19 (1993).

15. H. Serrai, L. Senhadji, D.B. Clayton, Z. Chun, and R.E. Lenkinski, Water modeled signal removal and data quantification in localized MR Spectroscopy using a time-scale postacquisition method, J. Magn. Reson. 149, 45-51 (2001).

16. A.A. Maudsley, Spectral lineshape determination by self-deconvultion, J. Magn. Reson.B 106(1), 47-57 (1995). 
17. S. Mori, S.M. Eleff, U. Pilatus, N. Mori, and P.C.M. Van Zijl, Proton NMR Spectroscopy of solvent-saturable resonances: A new approach to study $\mathrm{pH}$ effects in situ, Magn. Reson. Med. 40, 36-42 (1998). 


\section{$\underline{\text { Acknowledgements }}$}

Grant sponsors: NIH; Grant numbers; RO1CA70362, RO1MH49390, RO1NS31464 and RR20305. A part of this work was presented at the ninth scientific meeting and exhibition of ISMRM, April 21-27, 2001, Glasgow UK. 
Figures Captions:

Fig 1:

a) The single voxel spectrum of the phantom acquired without water suppression using the PRESS sequence at $3 \mathrm{~T}(\mathrm{TE}=35 \mathrm{~ms}$, TR $=2 \mathrm{sec}$, $2 \mathrm{~K}$ points, and $5000 \mathrm{~Hz}$ ). The arrows show the symmetric sidebands related to the water peak.

b) The spectrum of the modulus of the signal whose spectrum is shown in Fig.1a. The sidebands are removed. Note the symmetry of the metabolite peaks about the water peak.

c) The single voxel spectrum of the phantom acquired without water suppression using the PRESS sequence at $3 \mathrm{~T}(\mathrm{TE}=144 \mathrm{~ms}, \mathrm{TR}=2 \mathrm{sec}$, $2 \mathrm{~K}$ points, and $5000 \mathrm{~Hz}$ ). The arrows show the symmetric sidebands related to the water peak which decrease compared to those in Fig.1 a.

d) The spectrum of the modulus of Fig. 1c. The sidebands are removed.

Fig 2:

a) An expansion of the aliphatic part of the spectrum of the selected voxel from the white matter (2D-CSI PRESS, 16x16, 1 NEX, FOV= $24 \mathrm{~cm}$, $\mathrm{TR}=2 \mathrm{~s}, \mathrm{TE}=35 \mathrm{~ms}, 512$ points and $2000 \mathrm{~Hz}$ ). Note that the baseline shows a severe distortion, especially in the NAA region.

b) The aliphatic part of the spectrum of Fig 2a after removal of the sidebands. The baseline is corrected. 
Fig 3:

a) The water peak from the spectrum of Fig $2 b$.

b) The results obtained by fitting the water peak using spectral fitting methods.

c) The difference between (a) and (b).

Fig 4:

a) The region of NAA, creatine and choline from the spectrum of Fig $3 \mathrm{c}$ after water removal.

b) The results obtained by fitting the three peaks using spectral fitting methods.

c) The difference between (a) and (b). 
TABLE 1: Estimated and reference relative and absolute metabolite concentrations of the single voxel and 2D-CSI in-vitro and in-vivo data at TE $=35 \mathrm{~ms}$

\begin{tabular}{|c|c|c|c|c|c|c|}
\hline & NAA/creatine & Choline/creatine & $\begin{array}{c}\text { NAA } \\
(\mathrm{mmol} / \mathrm{kg}) \\
\end{array}$ & $\begin{array}{c}\text { creatine } \\
(\mathrm{mmol} / \mathrm{kg})\end{array}$ & $\begin{array}{c}\text { choline } \\
(\mathrm{mmol} / \mathrm{kg})\end{array}$ & $\begin{array}{c}\text { Water } \\
\text { suppression }\end{array}$ \\
\hline In-vitro (SV) & $1.30[1.25]$ & $0.88[\mathbf{0 . 9 0}]$ & I & l & l & yes \\
\hline In-vitro (SV) & 1.20 [1.25] & $0.96[\mathbf{0 . 9 0}]$ & $12.8[12.5]$ & $10.7[\mathbf{1 0}]$ & $3.4[3]$ & no \\
\hline In-vivo (SV) & 1.58 & 0.72 & l & l & l & yes \\
\hline In-vivo (SV) & 1.52 & 0.74 & $9.66[8.81]$ & $6.32[6.33]$ & $1.55[1.58]$ & no \\
\hline $\begin{array}{c}\text { In-vitro (2D- } \\
\text { CSI) }\end{array}$ & $1.26[1.25]$ & $0.93[\mathbf{0 . 9 0}]$ & l & l & l & yes \\
\hline $\begin{array}{c}\text { In-vitro (2D- } \\
\text { CSI) }\end{array}$ & $1.23[1.25]$ & $0.87[\mathbf{0 . 9 0}]$ & 13.9 [12.5] & $11.4[\mathbf{1 0}]$ & $3.3[3]$ & no \\
\hline $\begin{array}{c}\text { In-vivo (2D- } \\
\text { CSI) }\end{array}$ & 1.57 & 0.70 & l & l & / & yes \\
\hline $\begin{array}{c}\text { In-vivo (2D- } \\
\text { CSI) }\end{array}$ & 1.44 & 0.63 & $9.7[8.81]$ & $6.73[6.33]$ & 1.42 [1.58] & no \\
\hline
\end{tabular}

Note. The written values in bold are the reference values for the in-vitro data. The written values in italic bold are the reference values taken from the literature for the in-vivo white matter region (8). SV stands for single voxel. 
TABLE 2: Calculated creatine SNR (peak height) and width at half height (WHH) of the water peak before and after frequency demodulations.

\begin{tabular}{|c|c|c|c|c|c|}
\hline & $\mathrm{TE}(\mathrm{ms})$ & $\begin{array}{c}\text { SNR (peak } \\
\text { height)[before] }\end{array}$ & $\begin{array}{c}\text { SNR (peak } \\
\text { height)[after] }\end{array}$ & $\begin{array}{c}\text { WHH }(\mathrm{Hz}) \\
\text { [before] }\end{array}$ & $\begin{array}{c}\text { WHH (Hz) } \\
\text { [after] }\end{array}$ \\
\hline In-vitro (SV) & 35 & $10 / 1$ & $14 / 1$ & 3.3 & 2.8 \\
\hline In-vitro (SV) & 144 & $7 / 1$ & $10 / 1$ & 3.0 & 2.8 \\
\hline In-vitro (CSI) & 35 & 4/1. & $8 / 1$. & 3.6 & 2.7 \\
\hline In-vitro (CSI) & 144 & $3.5 / 1$ & $6 / 1$ & 2.7 & 2.7 \\
\hline In-vivo (SV) & 35 & $5 / 1$ & $6 / 1$ & 8.2 & 7.8 \\
\hline In-vivo (SV) & 144 & $4 / 1$ & $5 / 1$ & 7.9 & 7.8 \\
\hline In-vivo (CSI) & 35 & $7 / 1$. & $13 / 1$ & 8.8 & 7.6 \\
\hline In-vivo (CSI) & 144 & $4.5 / 1$ & $5 / 1$ & 7.8 & 7.6 \\
\hline
\end{tabular}

Note: SV stands for single voxel. Before $=$ Data without water suppression + FT. After $=$ Modulus of the data without water suppression $+\mathrm{FT}$. an $\mathrm{LB}=2 \mathrm{~Hz}$ was used. 


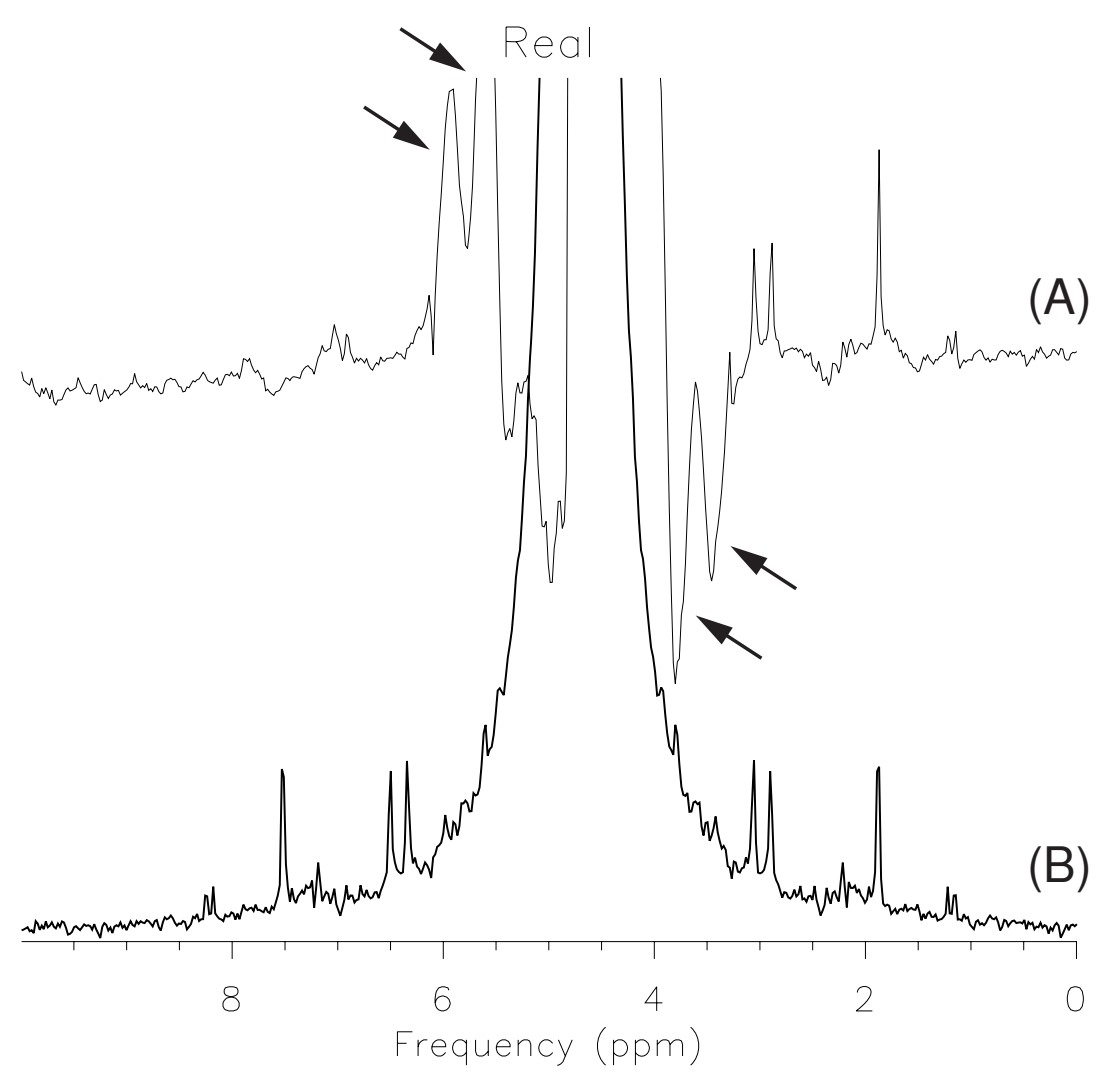


Real

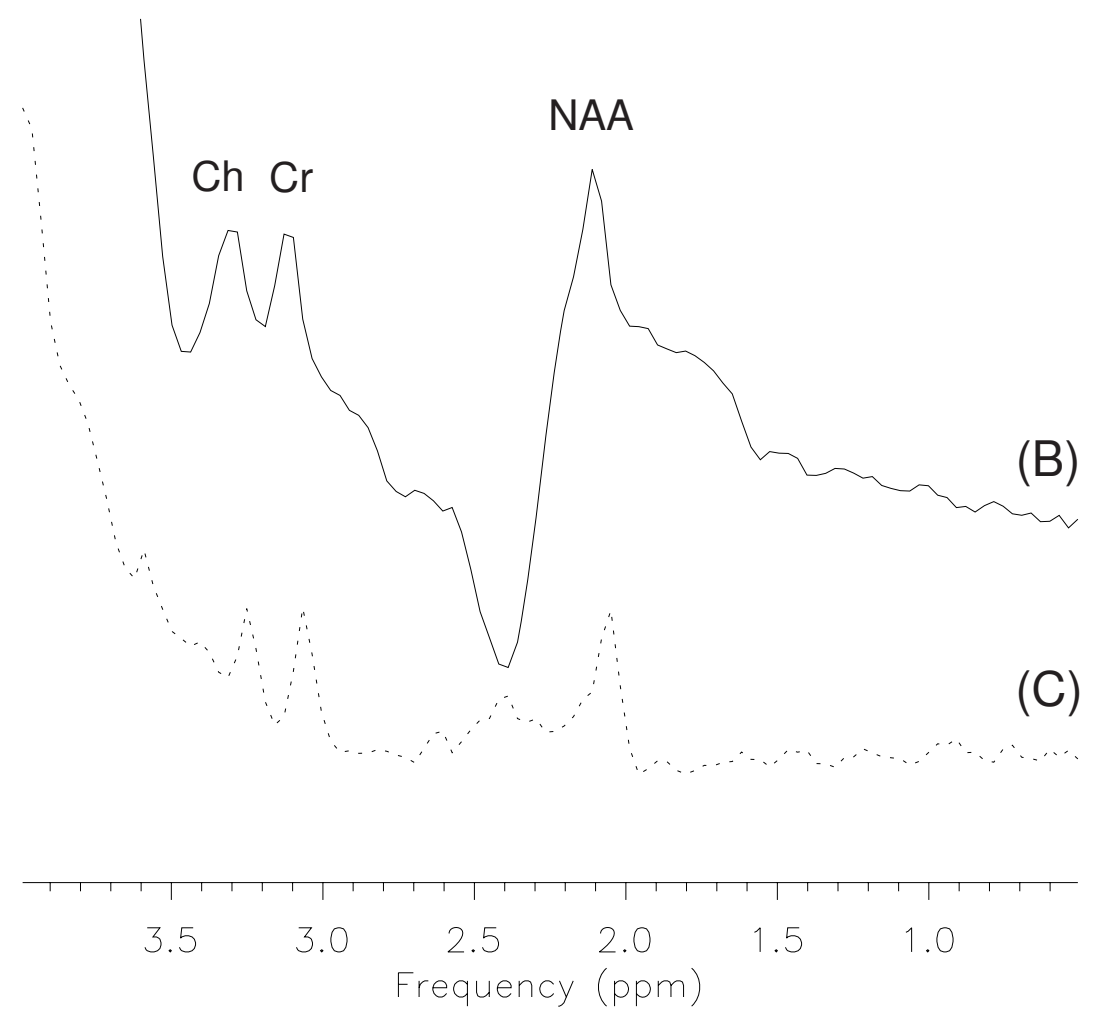




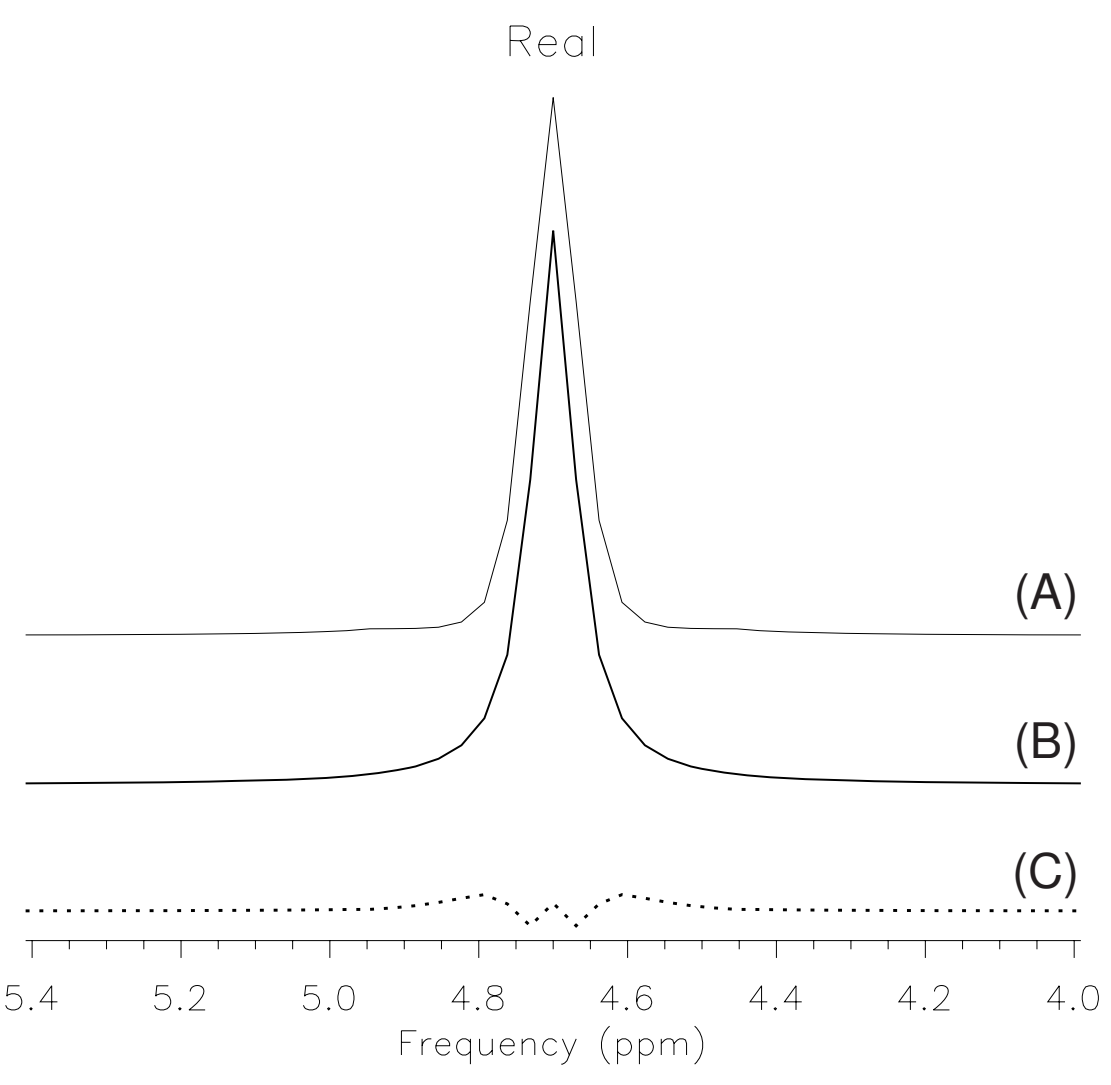




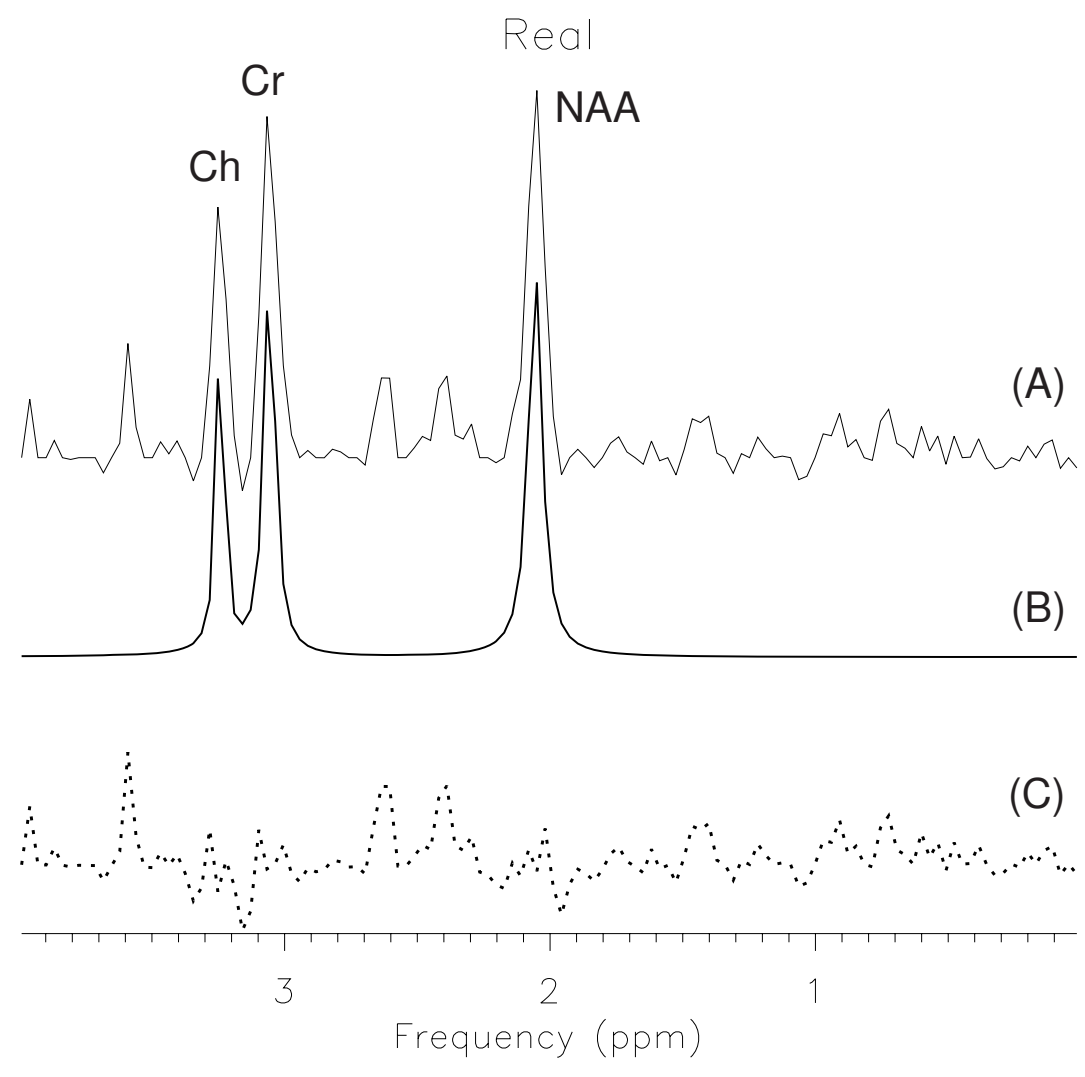

